\title{
Unilaterale Lungenvolumenreduktion mit intra- bronchialen Ventilen nach Selektion mittels endoskopischer Kollateralventilationsmessung
}

\author{
Unilateral Reduction of Lung Volume by Application of Intrabronchial Valves on \\ Patients Selected after Endoscopic Collateral Ventilation Assessment
}

Autoren

Institut
K.-J. Franke, G. Nilius, U. Domanski, K.-H. Rühle

Klinik für Pneumologie und Beatmungsmedizin, Helios Klinik Ambrock, Universität Witten/Herdecke, Lehrstuhl für Innere Medizin I, Hagen eingereicht 24.9.2013 akzeptiert nach Revision 7.11.2013

Bibliografie

DOI http://dx.doi.org/ 10.1055/s-0033-1359025 Online-Publikation: 16.12.2013 Pneumologie 2014; 68: 100-105 (c) Georg Thieme Verlag KG Stuttgart · New York ISSN 0934-8387

Korrespondenzadresse Dr. Karl-Josef Franke Klinik für Pneumologie Helios Klinik Ambrock Ambrocker Weg 60 58091 Hagen karl-josef.franke@helioskliniken.de

\section{Zusammenfassung \\ V}

Hintergrund: Mittels endoskopischer Kollateralventilationsmessung (ECVA) können Emphysempatienten für eine Ventilanlage zur Lungenvolumenreduktion selektiert werden. Wünschenswert ist eine post interventionell resultierende, klinisch relevante Verbesserung der körperlichen Leistungsfähigkeit der selektierten Klientel.

Methode: Patienten mit einer fortgeschrittenen COPD wurden einem Screening zur Lungenvolumenreduktion unterzogen. Selektierte Patienten erhielten eine ECVA, bei Patienten mit einem Anstieg des Kollateralwiderstandes Rkoll auf $>10 \mathrm{cmH}_{2} \mathrm{O} /(\mathrm{ml} / \mathrm{s})$ innerhalb einer Messdauer von bis zu $300 \mathrm{~s}$ wurden unilateral lobär intrabronchiale Ventile (IBV) platziert. Die Differenzen von Residualvolumen (RV) und Gehstrecke im 6-Minuten-Gehtest (6MWT) zwischen den Zeitpunkten TX (nach Ventilanlage) minus T1 (vor Ventilanlage) wurden errechnet.

Ergebnisse: Von 310 Emphysempatienten erfüllten 17 die Kriterien zur ECVA, 10/17 eigneten sich zur Ventilanlage. Nach $312 \pm 170$ Tagen (TX-T1) betrug die Zunahme der Gehstrecke $44 \pm 89,7 \mathrm{~m}, 7 / 10$ Patienten profitierten klinisch relevant ( $>25 \mathrm{~m}$ ). Die RV-Differenz korrelierte mit der Gehstreckendifferenz, nicht aber mit der ECVA-Messdauer.

Schlussfolgerungen: Nur ein geringer Teil der Patienten mit einem fortgeschrittenen Lungenemphysem scheint für eine endoskopische Lungenvolumenreduktion mittels Ventilanlage in Frage zu kommen. Eine unilaterale lobäre IBVAnlage kann zu einer dauerhaften, klinisch relevanten Verbesserung der körperlichen Leistungsfähigkeit führen. Die ECVA-Messdauer sollte zumindest 300 s betragen.

\section{Abstract \\ V}

Background: Endoscopic collateral ventilation assessment (ECVA) can be used to select patients suffering from emphysema for application of valves to achieve a reduction of lung volume. The objective is to obtain a post-operatively minimal clinically important improvement of the selected patient's physical capacity.

Methods: Patients with severe chronic obstructive pulmonary disease (COPD) underwent examination for a possible reduction of lung volume. Those selected got ECVA. Patients whose collateral resistance (Rcoll) increased to Rcoll $>10 \mathrm{~cm} \mathrm{H}_{2} \mathrm{O} /(\mathrm{mL} / \mathrm{s})$ within a measurement duration of up to $300 \mathrm{~s}$, were submitted to unilateral lobar application of intrabronchial valves (IBV). Differences in residual volume (RV) as well as distances in 6 minute walk tests (6MWT) between the time after application of valves (TX) and that before (T1) were calculated (TX minus T1).

Results: Of a tested 310 emphysema patients, 17 met criteria allowing ECVA; 10 of 17 proved eligible for valve application. After $312 \pm 170$ days (TX-T1) the increase in walking distance was $44 \pm 89.7 \mathrm{~m} ; 7$ of 10 patients achieved clinically relevant improvements (>25 m). Differences in $\mathrm{RV}$ proved to correlate with differences in walking distances, but not yet with differences in measurement duration in ECVA.

Conclusions: Only a small portion of patients with progressive emphysema of the lung seem suitable for endoscopic reduction of lung volume by application of valves. Unilateral lobar application of IBV can result in durable, clinically relevant improvements of the patient's physical capacity. ECVA measurements should take a minimum of $300 \mathrm{~s}$. 


\section{Einleitung}

Zur Behandlung des fortgeschrittenen heterogenen Lungenemphysems besteht die Möglichkeit, endoskopisch Einweg-Ventile in den Atemwegen zu platzieren [1 - 3]. Ziel sollte eine unilaterale, vollständige Okklusion der zentralen Atemwege des am stärksten destruierten Lungenlappens sein, um derart eine lobäre Atelektasenbildung zu erzeugen [4-5]. Gelingt eine relevante Lungenvolumenreduktion [6], kann in mehr als 50\% der Fälle mit einer klinisch relevanten Verbesserung von FEV1, körperlicher Leistungsfähigkeit und krankheitsbezogener Lebensqualität gerechnet werden [7]. Möglicherweise senkt das Erreichen einer relevanten Lungenvolumenreduktion darüber hinaus das Mortalitätsrisiko $[8,9]$. Eine Erhöhung der täglichen physischen Aktivität des Patienten als Folge einer verlängerten Gehstrecke im 6Minuten-Gehtest könnte hierfür eine Erklärung sein [10], allerdings scheint im Vergleich zur FEV1 gerade die körperliche Leistungsfähigkeit weniger dauerhaft post interventionell verbessert zu sein $[4,7]$.

Zwei unterschiedlich gebaute Ventile, „endobronchiales Ventil“ (EBV) bzw. „intrabronchiales Ventil“ (IBV) benannt, sind implantierbar; zur Wirksamkeit eines unilateralen lobären Verschlusses mit dem IBV liegen bisher jedoch kaum Daten vor [11].

Darüber hinaus ist unbekannt, wie viele Patienten mit einer fortgeschrittenen COPD für eine endoskopische Lungenvolumenreduktion mittels Ventilanlage überhaupt infrage kommen.

Post-Hoc-Analysen zeigen, dass Voraussetzung einer relevanten Volumenminderung des Ziellappens das Fehlen einer interlobären kollateralen Ventilation zwischen Ziellappen und benachbarten Lungenlappen ist [4,7]. Aufgrund kollateraler Luftströme in der emphysematös destruierten Lunge käme es zu einer Wiederbelüftung des Ziellappens. Surrogatparameter einer fehlenden kollateralen Ventilation in den zitierten Studien war die Integrität des Lappenspaltes in der hochauflösenden Computertomografie (HRCT). Eine funktionelle Methode, das Fehlen einer interlobären kollateralen Ventilation und damit eine Volumenminderung des Ziellappens nach Ventilanlage vorauszusagen, ist die endoskopische Kollateralventilationsmessung (ECVA, endoscopic collateral ventilation assessment). Mithilfe eines im Lappenbronchus des Ziellappens platzierten Ballonkatheters wird der Kollateralwiderstand Rkoll errechnet, wobei ein Rkoll $<10 \mathrm{cmH}_{2} \mathrm{O} /(\mathrm{ml} / \mathrm{s})$ eine kollaterale Ventilation anzeigt, ein Rkoll $>10 \mathrm{cmH}_{2} \mathrm{O} /(\mathrm{ml} / \mathrm{s})$ das Fehlen einer solchen [12]. Die Voraussagegenauigkeit einer relevanten Volumenminderung beträgt 75\% [13-14]. Die notwendige Messdauer, um eine Voraussage treffen zu können, wird mit 2,5 Minuten angegeben, ist aber bisher nicht ausreichend untersucht [12-13].

Ein bodyplethysmografisch gemessenes, hohes Residualvolumen kann einerseits als Selektionskriterium für eine endoskopische Lungenvolumenreduktion gelten und vermag andererseits eine durch Ventileinlage bedingte Volumenminderung anzuzeigen [4,6-7,15]. Primäres Ziel der vorliegenden Fallstudie war die Änderung von Residualvolumen (RV) und Gehstrecke im 6Minuten-Gehtest (6MWT) als Maß für die körperliche Leistungsfähigkeit nach unilateraler lobärer Implantation von IBV. Vorherig wurden die Patienten selektiert mittels ECVA aus einer im Rahmen der klinischen Routine selektierten Klientel von COPDPatienten. Sekundär sollte die Korrelation von RV-Änderung und Änderung der Gehstrecke im 6MWT sowie die Korrelation von RV-Änderung und Messdauer in der ECVA bis zum Erreichen eines relevanten Rkoll $>10 \mathrm{cmH}_{2} \mathrm{O} /(\mathrm{ml} / \mathrm{s})$ errechnet werden.

\section{Methodik}

\section{7}

Die Grundsätze der Helsinki Deklaration 1975 (1983) wurden beachtet. Die Zustimmung der Ethikkommission der Universität Witten/Herdecke zur Datenanalyse liegt vor.

\section{Patienten}

Patienten mit einer fortgeschrittenen COPD, welche im Zeitraum 1/11 -6/12 stationär behandelt wurden und die trotz rehabilitativer Maßnahme und stadiengerechter medikamentöser Therapie einen erheblichen Leidensdruck aufgrund einer reduzierten körperlichen Belastbarkeit angaben, wurden einem Screening zur Lungenvolumenreduktion unterzogen. Dieses Screening wurde 2010 für die klinische Routine standardisiert:

Alle Patienten werden in einer interdisziplinären Emphysemkonferenz vorgestellt, für die Auswahl zur möglichen Ventilanlage muss eine chirurgische Lungenvolumenreduktion ausgeschlossen worden sein.

Folgende Einschlusskriterien für eine IBV-Anlage werden zugrunde gelegt:

RV > 200\% Soll; Gehstrecke (D) im 6MWT: $300 \mathrm{~m}>\mathrm{D} \geq 100 \mathrm{~m}$. Ein fortgeschrittenes heterogenes Lungenemphysem wird von 2 Fachärzten aus den Bereichen Pneumologie sowie Radiologie unabhängig voneinander mittels HRCT diagnostiziert, wobei auf einer Skala von 0 bis 3 ( 0 = homogenes Emphysem, 3 = ausgeprägt heterogen) beide Untersucher die Heterogenität mit 2 oder 3 bezeichnen müssen. Als Zielzone wird der in der HRCT am stärksten destruierte Lappen gewählt; außerdem wird zur Detektion des Ziellappens die Perfusionsszintigrafie zu Hilfe genommen mit Bestimmung des am geringsten perfundierten Lungenareals. Ausschlusskriterien sind Bronchiektasen und eine vermehrte Sekretproduktion, eine akute Exazerbation, eine instabile kardiale Begleiterkrankung, Emphysembullae > 1/3 des Lappenvolumens.

\section{Endoskopische Kollateralventilationsmessung (ECVA)}

Bei Erfüllung der Einschlusskriterien für eine IBV-Anlage wurden Patienten einer flexiblen Bronchoskopie mit ECVA (Chartis Pulmonary Assessment System, Pulmonx, Redwood) unterzogen. Die Bronchoskopie erfolgte am spontan atmenden Patienten unter Sedierung mit Midazolam und Propofol. Der Ablauf der ECVA wurde bereits beschrieben [12-14]. Wurde innerhalb einer Messdauer $\leq 300 \mathrm{~s}$ ein Rkoll $>10 \mathrm{cmH}_{2} \mathrm{O} /(\mathrm{ml} / \mathrm{s})$ errechnet, wurde dies im Sinne einer nicht relevanten interlobären kollateralen Ventilation gewertet und Patienten wurden zur IBV-Anlage vorgesehen.

\section{Ventilanlage}

Eine schriftliche Einverständniserklärung zu Bronchoskopie und Ventilanlage sowie eine Datenschutzerklärung wurden von den Patienten eingeholt. Die IBV-Anlage (Olympus Medical Co) erfolgte in Vollnarkose im Rahmen einer starren Bronchoskopie. Der Ablauf der IBV-Anlage wurde bereits beschrieben [16-17].

\section{Zielparameter}

Die Änderungen von RV und Gehstrecke im 6MWT wurden errechnet zwischen den Zeitpunkten T1 und TX. T1 bezeichnet einen Zeitpunkt $\leq 24 \mathrm{~h}$ vor Ventilanlage, TX bezeichnet den letzten erfassten Zeitpunkt im Follow-up nach Ventilanlage. Patienten werden nach Ventilanlage standardisiert im Rahmen der klinischen Routine nachuntersucht. TX-T1 beträgt dabei $\geq 3$ Monate; werden die Ventile eines Patienten wieder entfernt, bezeichnet 
Tab. 1 Zielparameter IBV-Anlage ( $\mathrm{n}=10)$ : Änderung von Residualvolumen (RV) und Gehstrecke im 6-Minuten-Gehtest (6MWT) zwischen den Zeitpunkten T1 ( $\leq 24 \mathrm{~h}$ vor Ventilanlage) und TX ( $312 \pm 170$ Tage nach Ventilanlage) sowie Anteil Patienten mit klinisch relevanter Besserung: MCID (minimal clinical important difference) $R V>0,31 \mathrm{~L}$ und 6,1\% [10], 6MWT > $25 \mathrm{~m}$ [22].

\begin{tabular}{|lllll} 
& T1 & TX & Differenz TX-T1 & Patienten mit klinisch relevanter Besserung \\
\hline RV & $290,2 \pm 57,5 \%$ Soll & $256,9 \pm 58,1 \%$ Soll & $-33,3 \pm 64,8 \%$ Soll & 7 von 10 \\
\hline Gehstrecke 6MWT & $186,5 \pm 67,1 \mathrm{~m}$ & $230 \pm 94,7 \mathrm{~m}$ & $44 \pm 89,7 \mathrm{~m}$ & 7 von 10 \\
\hline
\end{tabular}

TX einen Zeitpunkt $\leq 24$ h vor Entfernung. Die Nachuntersuchung umfasst zumindest Anamnese und körperliche Untersuchung sowie eine Lungenfunktionsprüfung einschließlich 6MWT.

Der 6MWT erfolgte nach Standardkriterien [18-21]. Als klinisch relevante Änderung (minimal clinical important difference, MCID) des RV wurden eine Reduktion von 0,31 L und 6,1\% angesehen [6], bzgl. des 6MWT eine Zunahme der Gehstrecke von mindestens $25 \mathrm{~m}$ [22].

\section{ECVA-Messdauer und Korrelationen}

Die Dauer der ECVA-Messung bis zum Erreichen eines Rkoll $>10 \mathrm{cmH}_{2} \mathrm{O} /(\mathrm{ml} / \mathrm{s})$ wurde festgehalten. Die Korrelation von RVDifferenz (\%Soll) TX-T1 und Differenz der Gehstrecke im 6MWT TX-T1 wurde errechnet, zudem die RV-Differenz in Abhängigkeit vom Erreichen einer klinisch relevanten Gehstreckendifferenz (< bzw. > $25 \mathrm{~m}$ ). Die Korrelation von RV-Differenz und Messdauer der ECVA bis zum Erreichen eines relevanten Rkoll $>10 \mathrm{cmH}_{2} \mathrm{O}$ $(\mathrm{ml} / \mathrm{s})$ wurde errechnet, zudem die RV-Differenz in Abhängigkeit von einer ECVA-Dauer < oder $>150$ s.

\section{BODE-Index}

Unabhängig von den formulierten Zielen wurde zu den Zeitpunkten T1 und TX der BODE-Index erhoben (Body Mass Index, Obstruktion: FEV1, Dyspnoe: modified Medical Research Council, Exercise Capacity: 6MWT). Hierbei handelt es sich um ein multidimensionales (s. o.) und abgestuftes (10-Punkte-Skala, 4 RisikoQuartilen) Messsystem für COPD-Patienten, das zur Evaluation von krankheitsbeeinflussenden Interventionen eingesetzt werden kann. Als Ansprechen auf eine Intervention kann eine Reduktion um mindestens einen Punkt gewertet werden. Die klinische Antwort zeigt sich in einer Reduktion von Sterblichkeit und Hospitalisationsrate bzw. -dauer [23-27].

\section{Risiko}

Innerhalb der ersten 12 Monate nach endoskopischer Ventilanlage ist die Wahrscheinlichkeit von Komplikationen im Kontrollgruppenvergleich nicht signifikant erhöht [4, 7].

Für die von uns mit Ventilen behandelten Patienten haben wir für den Zeitraum TX-T1 folgende Daten erfasst: Anzahl Exazerbationen, Anzahl Pneumonien, Anzahl Pneumothoraces, Behandlungsdauer mit einer Pleuradrainage in Tagen, Anzahl Patienten mit Ventilentfernung.

\section{Statistik}

Deskriptiv erfolgten Berechnungen von Mittelwerten, Differenzen und Standardabweichungen aller zu den Zeitpunkten T1 und TX erhobenen Daten.

\section{Ergebnisse \\ $\nabla$}

310 Patienten wurden im Zeitraum 1/11 -6/12 dem Screening zur Lungenvolumenreduktion unterzogen. 17 von 310 Patienten erfüllten die Kriterien zur IBV-Anlage, diese 17 Patienten erhielten eine ECVA. Bei 7 von 17 Patienten wurde innerhalb einer Messdauer $\leq 300$ s ein Rkoll $<10 \mathrm{cmH}_{2} \mathrm{O} /(\mathrm{ml} / \mathrm{s})$, bei 10 von $17 \mathrm{~Pa}-$ tienten ein Rkoll $>10 \mathrm{cmH}_{2} \mathrm{O} /(\mathrm{ml} / \mathrm{s})$ errechnet; alle 10 Patienten mit einem Rkoll $>10 \mathrm{cmH}_{2} \mathrm{O} /(\mathrm{ml} / \mathrm{s})$ erhielten eine unilaterale lobäre IBV-Anlage (Alter $66 \pm 7$ Jahre, BMI 22,7 \pm 4 ).

Es wurden 4,8 $\pm 0,9$ Ventile pro Patient implantiert, 5-mal wurde der rechte und 1-mal der linke Oberlappen behandelt, 1-mal der rechte und 3-mal der linke Unterlappen. War die Seitenwahl nach CT-morphologischen Kriterien uneindeutig, konnte die Perfusionsszintigrafie ausschlaggebend sein für die Festlegung des Ziellappens.

Die Zeitspanne TX-T1 betrug $312 \pm 170$ Tage (range $84-581$ ). Bei 2 Patienten wurden die Ventile im Verlauf wieder entfernt, in einem Fall auf Wunsch des Patienten wegen fehlender subjektiver Besserung (TX-T1 84 Tage), im anderen Fall wegen wiederholter Exazerbationen (TX-T1 271 Tage).

\section{Zielparameter $(\bullet$ Tab. 1$)$}

Das RV \%Soll betrug zum Zeitpunkt T1 290,2 $\pm 57,5$, zum Zeitpunkt TX 256,9 $\pm 58,1$. Im Verlauf TX-T1 zeigte sich eine Reduktion des RV von 33,3 $\pm 64,8 \%$ Soll bzw. absolut von $0,6 \pm 1,3 \mathrm{~L}$, entsprechend einer Reduktion um 7,6 $\pm 21,0 \%$.

Die Gehstrecke im 6MWT betrug zum Zeitpunkt T1 186,5 $\pm 67,1$ m, zum Zeitpunkt TX 230 $\pm 94,7$ m. Im Verlauf TX-T1 zeigte sich eine Zunahme der Gehstrecke von $44 \pm 89,7 \mathrm{~m}$.

Eine klinisch relevante Reduktion (MCID) des RV ist für 7 von 10 Patienten und eine klinisch relevante Zunahme der Gehstrecke im 6MWT für ebenfalls 7 von 10 Patienten zu verzeichnen.

\section{ECVA-Messdauer und Korrelationen}

Die Messdauer der ECVA bis zum Erreichen eines Rkoll $>10 \mathrm{cmH}_{2} \mathrm{O} /(\mathrm{ml} / \mathrm{s}$ ) betrug 168,0 $\pm 82,2 \mathrm{~s}$ (range 70-300). Die RVDifferenz (\%Soll) TX-T1 korrelierte mit der Gehstreckendifferenz im 6MWT, bei Reduktion des RV zeigte sich eine klinisch relevante Gehstreckenverbesserung ( $\bullet$ Abb.1). Die RV-Differenz korrelierte nicht mit der ECVA-Messdauer bis zum Erreichen eines relevanten Rkoll $>10 \mathrm{cmH}_{2} \mathrm{O} /(\mathrm{ml} / \mathrm{s})$, die RV-Reduktion war unabhängig von einer ECVA-Dauer < oder >150 s ( Abb. 2).

\section{BODE-Index}

Im Zeitraum TX-T1 verbesserte sich der BODE-Index um 0,9 $\pm 1,2$ Punkte. Um mindestens einen Punkt reduzierte sich der BODEIndex nach Ventilanlage bei 6 von 10 Patienten. Vor Ventilanlage befanden sich 8 Patienten in der vierten und 2 in der dritten Quartile, in eine niedrigere Risiko-Quartile (von Quartile 4 in Quartile 3) gelangten 2 von 10 Patienten. 


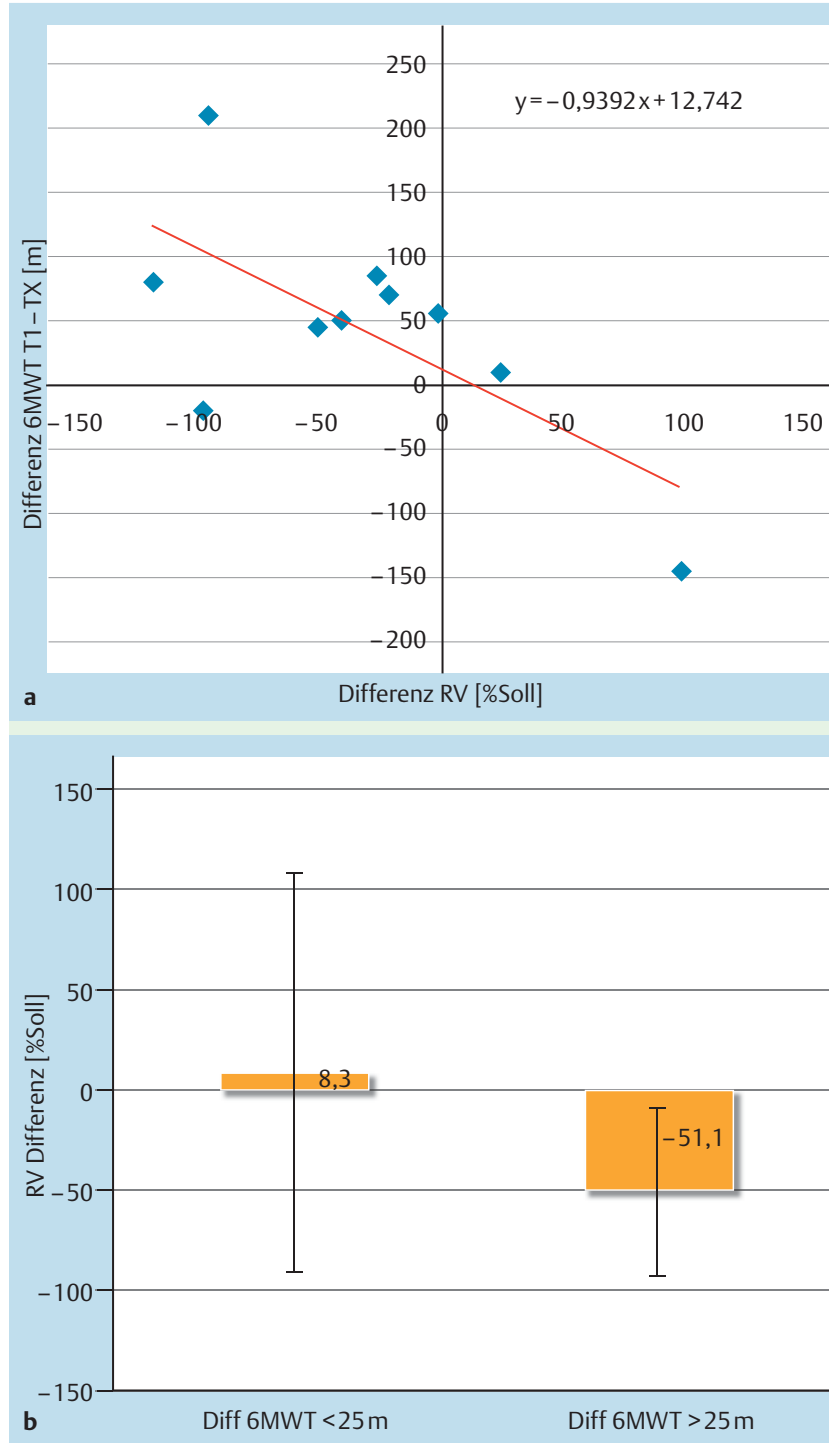

Abb. 1 a Korrelation von Gehstreckendifferenz TX-T1 im 6-MinutenGehtest (6MWT) versus Residualvolumen-Differenz (RV-Differenz). b RV-Differenz in Abhängigkeit vom Erreichen einer klinisch relevanten Gehstreckendifferenz (< bzw. > $25 \mathrm{~m}$ ). Eine Verbesserung der körperlichen Leistungsfähigkeit scheint abhängig von einer relevanten Volumenminderung zu sein.

\section{Risiko}

Im Zeitraum TX-T1 erlitten 4 Patienten insgesamt 6 Exazerbationen; in keinem Fall trat eine Pneumonie auf; 2 Patienten erlitten einen Pneumothorax, die Pleuradrainagen-Behandlungsdauer betrug insgesamt 9 Tage, in einem Fall wurde zur Pneumothorax-Behandlung 1 Ventil entfernt und nach einem Monat erneut implantiert, woraufhin sich erneut eine Atelektase im Ziellappen ausbildete.

\section{Diskussion \\ $\nabla$}

\section{Patienten}

Lediglich 5,5\% (17/310) der von uns gesichteten Patienten mit einer fortgeschrittenen COPD erfüllten die Einschlusskriterien für eine Ventilanlage bzw. zur Kollateralventilationsmessung. Sciurba et al. wählten mit 32,9\% (321/977) der gesichteten Pa-

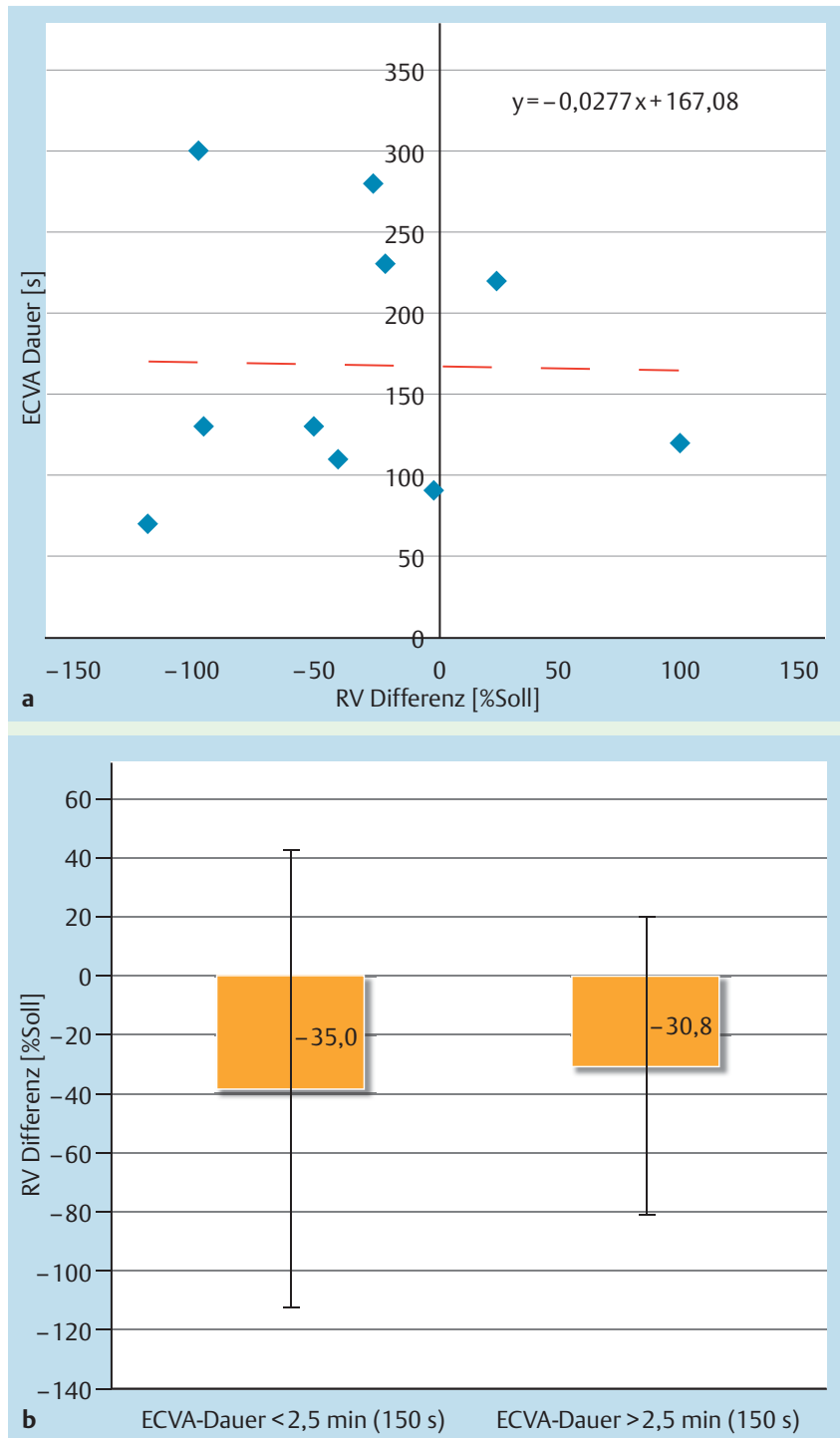

Abb.2 a Korrelation von Messdauer der endoskopischen Kollateralventilationsmessung (ECVA-Dauer) bis zum Erreichen eines relevanten Rkoll $>10 \mathrm{cmH}_{2} \mathrm{O} /(\mathrm{ml} / \mathrm{s})$ und Residualvolumen-Differenz (RV-Differenz) TX-T1. b RV-Differenz in Abhängigkeit von einer ECVA-Dauer< oder > 150 s. Bis zu einer Messdauer von 300 s scheint eine relevante Volumenminderung von dieser unabhängig zu sein.

tienten einen größeren Anteil als zur Randomisation geeignet aus, wobei aber z.B. der Grad der Heterogenität des Emphysems kein primäres Auswahlkriterium bedeutete [4]. Diesbezüglich wurden 14 unserer Patienten, die im übrigen die Einschlusskriterien erfüllten, aufgrund eines homogenen Emphysems ausgeschlossen. Nichtsdestotrotz weisen die Zahlen darauf hin, dass in der klinischen Routine möglicherweise ein nur geringer Teil der therapiebedürftigen Klientel mit fortgeschrittener Erkrankung letztlich für eine endoskopische Lungenvolumenreduktion in Frage kommt.

Von uns wurden nach ECVA aufgrund einer postulierten interlobären kollateralen Ventilation 7/17 Patienten $(41,2 \%)$ nicht mit Ventilen behandelt. Diese wären eventuelle Kandidaten für eine andere endoskopische Technik der Emphysembehandlung, wobei für diese Techniken Menge und Validität der bisher publizierten Daten geringer ist $[15,28]$. Mit 60,4\% war der Anteil von Patienten mit einer postulierten kollateralen Ventilation in einer 
anderen Arbeit höher als in der unsrigen, im Gegensatz zu der von uns durchgeführten prädiktiven funktionellen Methode der ECVA handelte es sich in der bezeichneten Studie allerdings um eine bildmorphologische Post-Hoc-Analyse von HRCT-Schichten [7]. Eine zusätzliche Beurteilung des betreffenden Lappenspaltes in der HRCT erfolgte durch uns nicht.

\section{Wirksamkeit}

Wir wählten für unsere Analyse als Zielparameter die Gehstrecke im 6MWT, da der größte subjektive Leidensdruck der Patienten häufig in einer Reduktion der körperlichen Leistungsfähigkeit besteht. Auch korreliert die Dauer eines täglichen körperlichen Trainings in Form von Gehen oder Radfahren sehr stark mit der Lebenserwartung des COPD-Patienten [10]. 7 von 10 unserer Patienten zeigten $312 \pm 170$ Tage nach IBV-Anlage eine klinisch relevante Verbesserung der Gehstrecke im 6MWT; bei Patienten mit intakter interlobärer Fissur sowie komplettem Verschluss des Ziellappens mit EBV fand sich nach 12 Monaten eine vergleichbare klinisch relevante Verbesserung der Gehstrecke in 10 von 18 Fällen [7]. Während in unserer Arbeit die mittlere Gehstreckenverbesserung $44 \pm 89,7 \mathrm{~m}$ betrug, weisen die einzigen anderen publizierten Daten zu einer unilateralen lobären IBV-Anlage nach 3 Monaten eine ähnliche Änderung der Gehstrecke bei ebenfalls 10 Patienten von 48,9 $\pm 53,0 \mathrm{~m}$ auf [11]. Letztgenannte Patienten waren zwar nicht explizit im Hinblick auf eine interlobäre kollaterale Ventilation selektiert, allerdings empirisch durch das vorherige Studium der HRCT ausgewählt worden.

Im Unterschied zur Literatur, in der sich vor Ventilanlage eine mittlere Gehstrecke > $300 \mathrm{~m}$ findet [4,7], ist für unsere Patienten ein schlechterer Ausgangsstatus mit 186,5 $\pm 67,1 \mathrm{~m}$ zu erwähnen. Da wir Patienten mit einer größeren Leistungseinschränkung untersuchten, konnte eine ausgeprägtere Leistungssteigerung erwartet werden. Insgesamt wird die klinische Relevanz der Leistungssteigerung unserer Patienten durch die postinterventionelle Verbesserung des BODE-Index um 0,9 $\pm 1,2$ Punkte untermauert.

Die genannte IBV-Studie zeigt im Mittel 3 Monate nach Ventilanlage mit $872 \pm 796 \mathrm{ml}$ eine relevante RV-Differenz, während in unserer Analyse diesbezüglich 7 von 10 Patienten klinisch relevant profitierten $[6,11]$. Darüber hinaus scheint eine hohe von uns errechnete Korrelation zwischen RV-Reduktion und Gehstreckenverbesserung ( $\bullet$ Abb.1) prinzipielle Überlegungen zur Wirksamkeit der Lungenvolumenreduktion durch Korrektur der Reduktion elastischer Rückstellkräfte und Optimierung der Arbeitslänge der Atemmuskulatur zu bestätigen [29].

\section{ECVA}

Mit einer Gehstreckenverbesserung von $44 \pm 89,7 \mathrm{~m}$ konnten wir die Ergebnisse einer weiteren Arbeit zur Selektion mittels ECVA bestätigen, welche 30 Tage nach Ventilanlage in der Gruppe der Patienten ohne interlobäre kollaterale Ventilation eine Gehstreckenverbesserung von $24 \pm 57$ m aufweist, wobei $45 \%$ der Patienten eine MCID von mindestens $26 \mathrm{~m}$ erreichten [14]. Die mittlere ECVA-Messdauer in unserer Klientel betrug 168,0 $\pm 82,2 \mathrm{~s}$, bei einer Messdauer bis zu 300 s war kein Unterschied im Hinblick auf eine relevante RV-Reduktion zu vermerken ( $\bullet$ Abb.2). Es ist durchaus vorstellbar, dass größere emphysematöse Lungenlappen weniger schnell eine exspiratorische Flussreduktion während der ECVA zeigen als kleinere Volumina. Bei fehlendem Anstieg des Rkoll $>10 \mathrm{cmH}_{2} \mathrm{O} /(\mathrm{ml} / \mathrm{s})$ sollte daher eine Voraussage erst mit einer ECVA-Messdauer von mindestens 300 s getroffen werden.

\section{Risiko}

In der Arbeit von Eberhardt et al. werden nach IBV-Anlage und einer Beobachtungszeit von 90 Tagen 4 Exazerbationen $(\mathrm{n}=22)$ berichtet, 2 Patienten erlitten darüber hinaus ein Atemversagen mit Beatmungspflichtigkeit, weshalb die Ventile in beiden Fällen wieder entfernt wurden [11]. Mit 6 Exazerbationen scheint in unserer Klientel die Zahl höher zu liegen, allerdings betrug der Follow-up-Zeitraum hier im Unterschied bei 9/10 Patienten zum Teil deutlich >90 Tage. Ein Patient erlitt allein 3 Exazerbationen, die Ventile wurden 271 Tage nach Anlage wieder entfernt.

Die Drainagedauer aufgrund eines Pneumothorax war mit 9 Tagen in unserer Klientel gegenüber 12 Tagen in o.g. Studie vergleichbar [11]. Aufgrund eines Pneumothorax haben wir in einem Fall für die Dauer eines Monats ein Ventil entfernt, woraufhin sich der atelektatische Lungenlappen zwar rasch wieder entfaltete und die Leckage dadurch aufgehoben wurde, jedoch klagte der Patient einhergehend mit eine Verschlechterung der Funktionsparameter deutlich vermehrt über Dyspnoe sowie eine Einschränkung der körperlichen Leistungsfähigkeit, ein Phänomen, das bereits beobachtet wurde [7].

\section{Schlussfolgerung}

Lediglich ein geringer Teil der Patienten mit einer fortgeschrittenen COPD und optimierter konservativer Therapie scheint für eine endoskopische Behandlung mit Ventilen geeignet zu sein. In einer selektierten Patientenklientel aber vermag eine unilaterale lobäre IBV-Anlage zu einer klinisch relevanten Verbesserung der körperlichen Leistungsfähigkeit zu führen, die auch länger als ein Jahr anhalten kann. Bei Verwendung der ECVA als Selektionsmethode kann auch nach mehr als 150 s Messdauer noch mit einem relevanten Anstieg des Kollateralwiderstandes gerechnet werden.

\section{Interessenkonflikt}

$\nabla$

K.-J. Franke erhielt von der Firma Olympus 2011 und 2012 ein Honorar für Vorträge im Rahmen einer Fortbildungsveranstaltung für Ärzte zur „Transbronchialen Nadelaspiration“.

G. Nilius, U. Domanski und K.-H. Rühle geben an, dass kein Interessenkonflikt besteht.

\section{Literatur}

1 Toma TP, Hopkinson NS, Hillier J et al. Bronchoscopic volume reduction with valve implants in patients with severe emphysema. Lancet 2003; 361: 931 - 933

2 Snell GI, Holsworth L, Borrill ZL et al. The potential for bronchoscopic lung volume reduction using bronchial prostheses: a pilot study. Chest 2003; 124: $1073-1080$

3 Venuta F, de Giacomo T, Rendina EA et al. Bronchoscopic lung-volume reduction with one-way valves in patients with heterogenous emphysema. Ann Thorac Surg 2005; 79: 411-416; discussion 416-417

4 Sciurba FC, Ernst A, Herth FJ et al. VENT Study Research Group. A randomized study of endobronchial valves for advanced emphysema. $\mathrm{N}$ Engl J Med 2010; 363: $1233-1244$

5 Ninane V, Geltner C, Bezzi M et al. Multicentre European study for the treatment of advanced emphysema with bronchial valves. Eur Respir J 2012; 39: 1319-1325

6 Hartman JE, Ten Hacken NH, Klooster K et al. The minimal important difference for residual volume in patients with severe emphysema. Eur Respir J 2012; 40: 1137-1141 
7 Herth FJ, Noppen M, Valipour A et al. International VENT Study Group. Efficacy predictors of lung volume reduction with Zephyr valves in a European cohort. Eur Respir J 2012; 39: 1334-1342

8 Hopkinson NS, Kemp SV, Toma TP et al. Atelectasis and survival after bronchoscopic lung volume reduction for COPD. Eur Respir J 2011; 37: $1346-1351$

9 Venuta F, Anile M, Diso D et al. Long-term follow-up after bronchoscopic lung volume reduction in patients with emphysema. Eur Respir J 2012; 39: $1084-1089$

10 Garcia-Aymerich J, Lange P, Benet $M$ et al. Regular physical activity reduces hospital admission and mortality in chronic obstructive pulmonary disease: a population based cohort study. Thorax 2006; 61: 772 778

11 Eberhardt R, Gompelmann D, Schuhmann $M$ et al. Complete unilateral vs partial bilateral endoscopic lung volume reduction in patients with bilateral lung emphysema. Chest 2012; 142: 900-908

12 Aljuri $N$, Freitag $L$. Validation and pilot clinical study of a new bronchoscopic method to measure collateral ventilation before endobronchial lung volume reduction. J Appl Physiol 2009; 106: 774-783

13 Gompelmann $D$, Eberhardt $R$, Michaud $G$ et al. Predicting atelectasis by assessment of collateral ventilation prior to endobronchial lung volume reduction: a feasibility study. Respiration 2010; 80: 419-425

14 Herth FJ, Eberhardt R, Gompelmann D et al. Radiological and clinical outcomes of using Chartis ${ }^{\mathrm{TM}}$ to plan endobronchial valve treatment. Eur Respir J 2013; 41: 302 - 308

15 Slebos DJ, Klooster K, Ernst A et al. Bronchoscopic lung volume reduction coil treatment of patients with severe heterogeneous emphysema. Chest 2012; 142: 574-582

16 Springmeyer SC, Bolliger CT, Waddell TK et al. IBV Valve Pilot Trials Research Teams. Treatment of heterogeneous emphysema using the spiration IBV valves. Thorac Surg Clin 2009; 19: 247-253, ix-X

17 Sterman DH, Mehta AC, Wood DE et al. IBV Valve US Pilot Trial Research Team. A multicenter pilot study of a bronchial valve for the treatment of severe emphysema. Respiration 2010; 79: 222-233
18 Redelmeier DA, Bayoumi AM, Goldstein RS et al. Interpreting small differences in functional status: the Six Minute Walk test in chronic lung disease patients. Am J Respir Crit Care Med 1997; 155: 1278-1282

19 ATS Committee on Proficiency Standards for Clinical Pulmonary Function Laboratories. ATS statement: guidelines for the six-minute walk test. Am J Respir Crit Care Med 2002; 166: 111-117

20 Brooks D, Solway S, Gibbons WJ. ATS statement on six-minute walk test. Am J Respir Crit Care Med 2003; 167: 1287

21 Verrill DE, Fox L, Moore JB et al. Validity and reliability of the North Carolina 6-minute cycle test. J Cardiopulm Rehabil 2006; 26: 224-230

22 Puhan MA, Chandra D, Mosenifar Z et al. National Emphysema Treatment Trial (NETT) Research Group. The minimal important difference of exercise tests in severe COPD. Eur Respir J 2011; 37: 784 - 790

23 Celli BR, Cote CG, Marin JM et al. The body-mass index, airflow obstruction, dyspnea, and exercise capacity index in chronic obstructive pulmonary disease. N Engl J Med 2004; 350: 1005-1012

24 Cote CG, Celli BR. Pulmonary rehabilitation and the BODE index in COPD. Eur Respir J 2005; 26: 630-636

25 Imfeld S, Bloch KE, Weder W et al. The BODE index after lung volume reduction surgery correlates with survival. Chest 2006; 129: 873-878

26 Martinez FJ, Han MK, Andrei AC et al. National Emphysema Treatment Trial Research Group. Longitudinal change in the BODE index predicts mortality in severe emphysema. Am J Respir Crit Care Med 2008; 178 : $491-499$

27 Puhan MA, Garcia-Aymerich J, Frey $M$ et al. Expansion of the prognostic assessment of patients with chronic obstructive pulmonary disease: the updated BODE index and the ADO index. Lancet 2009; 374: 704 711

28 Kramer MR, Refaely Y, Maimon $N$ et al. Bilateral endoscopic sealant lung volume reduction therapy for advanced emphysema. Chest 2012; 142 : $1111-1117$

29 Noppen M. Collateral ventilation in end-stage emphysema: a blessing or a curse for new bronchoscopic treatment approaches (or both)? Respiration 2007; 74: 493-495 